\title{
The influence of the cation type of ionic liquid on the production of nanocrystalline cellulose and mechanical properties of chitosan-based biocomposites
}

\author{
Aleksandra Grząbka-Zasadzińska (iD - Andrzej Skrzypczak · Sławomir Borysiak
}

Received: 24 July 2018/Accepted: 8 April 2019/Published online: 13 April 2019

(C) The Author(s) 2019

\begin{abstract}
In this paper three newly synthesized imidazolium ionic liquids with different structures of cations were used for hydrolysis of cellulose. It was found that type of the cation has a great influence on particle size and dispersity of produced cellulosic materials. The use of an ionic liquid with a cation with an aliphatic substituent resulted in filler characterized with wide dispersity range and some particles larger than $2 \mu \mathrm{m}$. Introduction of methyl group into imidazolium ring was responsible for production of particles below $350 \mathrm{~nm}$. Such particles were obtained also during hydrolysis of cellulose with ionic liquid containing a benzyl substituent with that difference that in this case particles had definitely narrower dispersity range. Moreover, type of the ionic liquid used affected crystallinity degree of the produced
\end{abstract}

celluloses. Produced cellulosic materials were used as fillers for chitosan matrix. A relationship between mechanical properties of polymer composites and the particle size as well as dispersity of cellulose, controlled by chemical modifications of cellulose with innovative ionic liquids was evaluated. Incorporation of filler with established dispersive properties made it possible to form composites characterized by the assumed properties, for example with high values of Young's modulus, tensile strength or elongation at break. Understanding the dependence between the structure of ionic liquids and dispersive properties of cellulose is an important step toward the design of composite materials with optimal mechanical properties.
Electronic supplementary material The online version of this article (https://doi.org/10.1007/s10570-019-02412-1) contains supplementary material, which is available to authorized users.
A. Grząbka-Zasadzińska $(\bowtie) \cdot$ A. Skrzypczak ·

S. Borysiak

Institute of Chemical Technology and Engineering,

Poznan University of Technology, Berdychowo 4,

60965 Poznan, Poland

e-mail: aleksandra.grzabka-zasadzinska@put.poznan.pl 


\section{Graphical abstract}
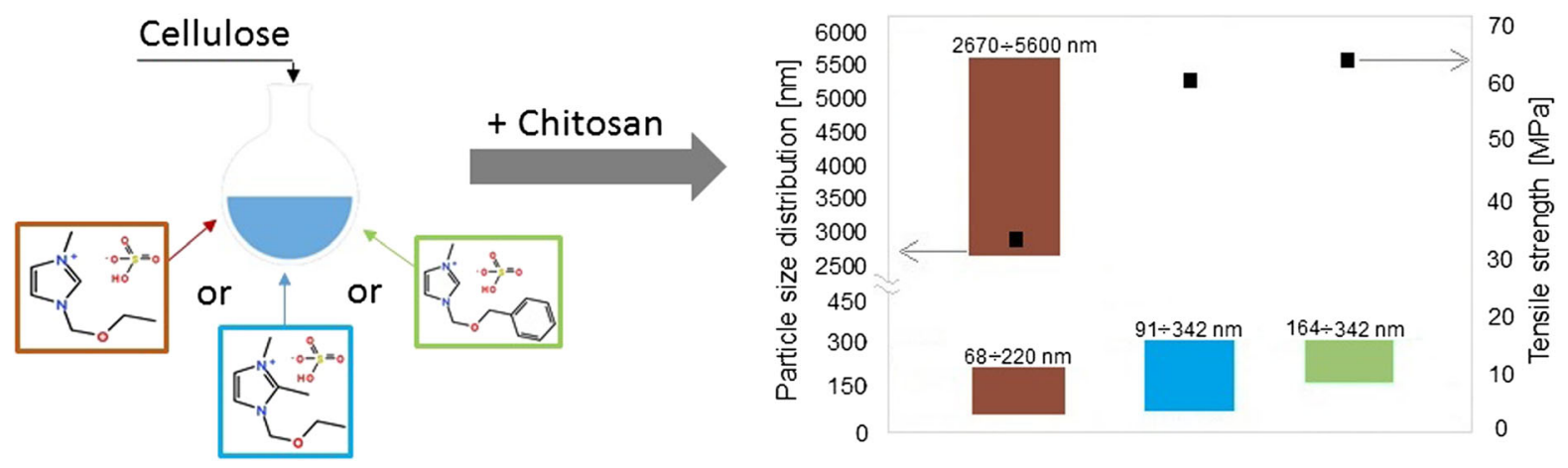

Keywords Nanocellulose - Ionic liquid · Chitosan · Composites

\section{Introduction}

Nanometric cellulose is currently a subject of very intensive scientific research. This is mainly due to the high availability and renewability of the cellulose. However, it is also caused by its attractive chemical and mechanical properties, which make it applicable in various fields. Nanometric cellulose combines the most important features of both cellulose materials and nanometric fillers (Trache et al. 2017). It is worth emphasizing that the type of the nanometric filler obtained from cellulose is particularly influenced by the origin of cellulose and methods of its chemical or mechanical treatment.

Nowadays, ionic liquids are used mainly to dissolve or modify biomass and cellulose (Brandt et al. 2013; Ghasemi et al. 2017; Borysiak et al. 2018), but there is also a growing interest in the use of ionic liquids for the direct production of nanometric cellulose. Swatloski et al. (2002) proved that cellulose can be dissolved, without any previous activation of pretreatment, in e.g. 1-butyl-3-methylimidazolium chloride and subsequently regenerated. Initially, it was claimed that the chloride anion, as a small acceptor of hydrogen bonds, in combination with 1-butyl-3methylimidazolium cation is the most effective in terms of dissolution of cellulose (Remsing et al. 2006). Liu et al. (2010) proved that interactions between acetate anions and hydroxyl groups of glucose are three times stronger than analogous interactions

between water and hydroxyl groups. Thus, it was confirmed that ionic liquids can effectively break H$\mathrm{O} \cdots \mathrm{H}-\mathrm{O}$ bonds. Over time, it turned out that ionic liquids containing halide anion are characterized by high viscosity, significantly limiting their applicability. In order to overcome this drawback, ionic liquids with formate, acetate and phosphate anions were used (Isik et al. 2014). Interestingly, the detailed mechanism of cellulose dissolution is still debatable. Nuclear magnetic resonance (NMR) and electron scattering studies have shown that the dissolution of cellulose in an ionic liquid is related to the presence of hydrogen bonds between cellulose hydroxyl protons and chloride ions of ionic liquid, while the role of cations was considered negligible (Remsing et al. 2006; Youngs 2011). Molecular dynamics studies on behavior of small bunch of cellulose in various ionic liquids showed that anions are intensely bound to hydroxyl groups on the cellulose surface, creating a negative electric charge that weakens the hydrogen bonds between the cellulose chains. Then, as a result of electrostatic attraction, the cations intercalate between the chains and cause them to separate (Li et al. 2015). Apart from the chemical structure of ionic liquid, one of the two main factors affecting the solubility of cellulose in ionic liquids is the crystallinity of cellulose. Crystalline regions are characterized by lower energy than amorphous regions, they are more energetically stable and, therefore the dissolution of the crystalline regions is more difficult (Lindman et al. 2010). In the initial stages the ionic liquid treatment of cellulose results in the elimination of amorphous regions from its structure, thus increasing the degree of crystallinity, similarly as in the case of acid hydrolysis (Jonoobi et al. 2015). The second factor responsible 
for dissolving cellulose is its hydrogen bonding system (Lindman et al. 2010) since breaking them is necessary to dissolve the polymer.

In accordance to the current state of knowledge, it seems that dissolving cellulose and production of nanometric cellulose requires the synergistic interaction of both, cations and anions of ionic liquids. There are many papers that prove that ionic liquids with different structures of cations have an ability to dissolve cellulose (Isik et al. 2014). Imidazole ionic liquids with $\mathrm{HSO}_{4}{ }^{-}$or $\mathrm{Cl}^{-}$anion were reported to be the most suitable for that purpose. It was also found that ionic liquids with these anions can be used in controlled hydrolysis of cellulose leading to formation of nanometric cellulose (Suzuki et al. 2014; Mao et al. 2015). However, just like in case of total dissolution of cellulose, there are no data regarding influence of the cation type of ionic liquid on production of nanometric cellulose. Therefore, finding an answer for this issue requires synthesis of new ionic liquids with planned size of cation.

Nanometric cellulose and chitosan are polysaccharides, which show a significant similarity in chemical structure. Introduction of nanometric cellulose into chitosan matrix results in obtaining biodegradable composites that combine unique features of both components. There are many papers regarding chitosan/nanometric cellulose composites (HPS et al. 2016) which are mainly used on a laboratory scale, but can also find application in the packaging (films for food products, paper coatings), chemical (catalysts, adsorbents) or in medicine (carriers of active substances) industry (Li et al. 2004; Fernandes et al. 2011; Dehnad et al. 2014; Rad-Moghadam and Dehghan 2014). It is known that the higher the L/D ratio of the filler, the better are its reinforcing properties (Pradhan et al. 2013). Comparison of mechanical properties obtained for chitosan filled with $10 \%$ of different types of nanometric cellulose (Fernandes et al. 2009; Khan et al. 2012; Rubentheren et al. 2016; Nandi and Guha 2018) showed that size of such filler is of great importance.

Up to now there is no literature data regarding influence of size of the cation of imidazole ionic liquid on mechanism of nanometric cellulose formation. It is of great importance to consider how the structure of ionic liquid can affect size and dispersity of produced cellulosic materials. It can be assumed that the design and synthesis of new ionic liquids, intended solely for production of nanometric cellulose, and not its complete dissolution, will constitute a significant scientific novelty. Determining the optimal cation structure of the ionic liquid and the determination of the process parameters using such a hydrolyzing agent would allow to control the quality of the obtained nanometric cellulose (size and dispersion of particles, degree of polydispersity, crystal structure). Finding this relationship is crucial in terms of production of chitosan composites with cellulose. Thus, the aim of this study was to define the influence of cellulose particle size obtained using newly synthesized imidazole ionic liquids on mechanical properties of chitosan composites.

\section{Experimental}

\section{Materials}

Cellulose Avicel PH-101 with average particle size of $50 \mu \mathrm{m}$ and high molecular weight chitosan from crab shells (degree of deacetylation 75-85\%) was purchased from Sigma-Aldrich. Acetic acid $80 \%$ purchased from POCH S.A. was diluted to $2 \%(\mathrm{v} / \mathrm{v})$ solution and used for composites formation.

All the reagents used for syntheses of new ionic liquids, i.e. 1-methylimidazole, 1-2-dimethylimidazole, chlormethyl-ethylether, formaldehyde, hydrochloric acid, sulfuric(VI) acid, benzyl alcohol, acetonitrile, heptane were purchased from Sigma-Aldrich in "for synthesis" quality or higher and were used without further purification or drying. Chlormethyl-benzylether was obtained by passing gaseous hydrochloric acid through a mixture of formaldehyde and benzyl alcohol, followed by two distillations under reduced pressure.

Synthesis of ionic liquids

Imidazolium ionic liquids were synthesized in a three stage process, according to methods which are described below.

The synthesis of acidic imidazolium ionic liquids consisted in three steps:

In the first step imidazolium chlorides were obtained in the reaction of 1-methylimidazole or 1,2-dimethylimidazole with chloromethylethyl ether (Scheme 1) or in the reaction of 1-methylimidazole with chloromethylbenzyl ether (Scheme 2).Chloromethylbenzyl ether was obtained by passing $\mathrm{HCl}$-gas through a mixture of formaldehyde and benzyl alcohol, according to the 
<smiles>[R]c1nccn1C</smiles><smiles>[R]c1n(C)cc[n+]1C[n+]1ccn(C)c1[R]</smiles>

$\mathrm{R}=\mathrm{H}, \mathrm{CH}_{3}$

Scheme 1 Synthesis of 3-ethoxymethyl-1-methylimidazolium hydrogen sulfate (emm) and 3-ethoxymethyl-1,2-dimethylimidazolium hydrogen sulfate (emd)

procedure described earlier (Urbanik et al. 1999). The final chloromethylbenzyl ether was twice distilled under reduced pressure. The yield was $68 \%$.

Preparation of 3-ethoxymethy-1-methyl- and 3ethoxymethyl-1,2-dimethylimidazolium chloride an anhydrous solution of $0.1 \mathrm{~mol}$ of 1-methylimidazole or 1,2-dimethylimidazole in acetonitrile was added to an anhydrous solution of $0.11 \mathrm{~mol}$ of chloromethylethyl ether, in acetonitrile. The reactions were carried out for $1 \mathrm{~h}$ at $320 \mathrm{~K}$ and then the solvent was evaporated in vacuum. Quaternization reaction products were purified by extraction with heptane at $343 \mathrm{~K}$. The yield of hygroscopic products: 3-ethoxymethyl-1methylimidazolium chloride and 3-ethoxymethyl-1,2dimethylimidazolium chloride was 94 and $91 \%$.

Preparation of 3-benzyloxymethy-1-methylimidazolium chloride an anhydrous solution of $0.1 \mathrm{~mol}$ of 1-methylimidazole in acetonitrile was added to an anhydrous solution of $0.11 \mathrm{~mol}$ of chloromethylbenzyl ether, in acetonitrile. The reaction was carried out for $2 \mathrm{~h}$ at $320 \mathrm{~K}$ and then the solvent was evaporated in vacuum. Quaternization reaction product was purified by extraction with heptane at $343 \mathrm{~K}$. The yield of hygroscopic product: 3-benzyloxymethy-1-methylimidazolium chloride was $85 \%$.Chloromethylethyl ether and chloromethylbenzyl ether readily hydrolyzed in a presence of small amount of water to form $\mathrm{HCl}$, which in turn gives the 1-methylimidazolium hydrochloride. The separation of the quaternization product and 1-methylimidazolium hydrochloride is practically impossible. For this reason, quaternization reactions with chloromethylethyl ether and chloromethylbenzyl ether were conducted under strictly anhydrous conditions. In the second step, the chloride anions in obtained imidazolium chlorides were exchanged to hydroxide anions, on the ion exchange resin (Dowex Monosphere 550 A UPW OH form resin) (Schemes 1, 2).

Preparation of imidazolium hydroxide $0.1 \mathrm{~mol}$ of imidazolium chloride was dissolved in water and passed through the column filled with Dowex Monosphere 550 A UPW OH form resin. In the third step so obtained imidazolium hydroxides were immediately reacted with sulfuric acid and appropriate imidazolium hydrogen sulfate was achieved (Schemes 1,2). Water was evaporated in vacuum.<smiles>Cn1ccnc1</smiles><smiles>Cn1cc[n+](COCc2ccccc2)c1</smiles>

Scheme 2 Synthesis of 3-benzyloxymethyl-1-methylimidazolium hydrogen sulfate (bom) 
Three imidazolium hydrogen sulfates were synthesized: 3-ethoxymethyl-1-methylimidazolium hydrogen sulfate (emm),3-ethoxymethyl-1,2-dimethylimidazolium hydrogen sulfate (emd), and 3-benzyloxymethyl-1-methylimidazolium hydrogen sulfate (bom). The total yield was $98.9 \%, 99 \%$, and $99.3 \%$.

In Table 1 chemical structures, names, and abbreviations of newly synthesized ionic liquids are given.

Hydrolysis of cellulose with ionic liquids

Micrometric cellulose I was mixed with tenfold excess of ionic liquid, the mixture was kept in $50{ }^{\circ} \mathrm{C}$ for $2 \mathrm{~h}$. Subsequently distilled water was added so that ionic liquid to water ratio was 80:20 (w/w). The mixture was heated up to $90{ }^{\circ} \mathrm{C}$ and the reaction was continued for next $6 \mathrm{~h}$ under constant stirring at $600 \mathrm{rpm}$. After that time hydrolysis was stopped by adding water. The obtained suspension was neutralized with significant amounts of water until $\mathrm{pH} \approx 7$ was reached, then sedimented, centrifuged, and filtered. Produced CNC was dried at $70{ }^{\circ} \mathrm{C}$ until constant weight (Binder FD series 23). After hydrolysis process ionic liquid was recovered using vacuum evaporator (Rotavapor RII, Büchi Labortechnik AG, at $70{ }^{\circ} \mathrm{C}, 82$ mbar, $24 \mathrm{~h}$ ). Water content in ionic liquid after recovery was $8 \%$ (w/w). The abovementioned procedure was applied for hydrolysis of cellulose I with each of ionic liquids. The yield of CNC production was in range from 65 to $80 \%$. Loss of the material was caused by the multi-step process of preparation of filler.
Preparation of composites

Chitosan/nanocrystalline cellulose composites were produced by solvent casting method described also by other authors (Dehnad et al. 2014; Falamarzpour et al. 2017). Firstly, chitosan was dissolved in $2 \%$ (v/v) $\mathrm{CH}_{3} \mathrm{COOH}$. Secondly, suspension of $\mathrm{CNC}$ (containing $1 \%, 3 \%$, and $5 \%(\mathrm{w} / \mathrm{w})$ of $\mathrm{CNC}$, in relation to dry mass of chitosan) in acetic acid was slowly added to chitosan solution, stirred for $5 \mathrm{~min}$ at $3000 \mathrm{rpm}$, and then homogenized using ultrasonic bath $(30 \mathrm{~min}$ at $40 \mathrm{kHz}, 100 \%$ power without heating). Mixtures were applied on Petri dishes and dried for $12 \mathrm{~h}$ at $35^{\circ} \mathrm{C}$. Obtained films were homogenous, no holes or big agglomerates were present.

Samples were named for ease of experiments and film characterization such as $\mathrm{CHT} / 5 \mathrm{CNC}$-bom where stands for chitosan composite with $5 \%$ loading of nanocrystalline cellulose I produced using 3-(benzyloxymethyl)1-methylimidazolium hydrogen sulfate(VI).

\section{NMR spectroscopy}

Chemical structure and purity of ionic liquids was confirmed using NMR spectroscopy. ${ }^{13} \mathrm{C}$ NMR and ${ }^{1} \mathrm{H}$ NMR spectra were recorded using Brucker Avance spectrometer at $600 \mathrm{MHz}$ and $150 \mathrm{MHz}$ frequency, respectively for ${ }^{1} \mathrm{H}$ NMR and ${ }^{13} \mathrm{C}$ NMR. Tetramethylsilane was used as an internal standard.

The results of NMR measurements are as follows:

Table 1 Structures and names of used ionic liquids

3-etoxymethyl-1,2-dimethylimidazolium hydrogen sulfate(VI)


3-(etoxymethyl)-1-methylimidazolium hydrogen sulfate(VI) (emm): ${ }^{1} \mathrm{H} \quad \mathrm{NMR} \quad(600 \mathrm{MHz}$, temp. $298 \mathrm{~K}$, DMSO-d $\left.\mathrm{d}_{6}, \mathrm{TMS}\right): \delta(\mathrm{ppm})=1.11-1.13(\mathrm{t}$, $\mathrm{J}=7,1 \mathrm{MHz}, 3 \mathrm{H})$; 3.55-3.58(q, J = 7.1 MHz, 2H); 3.91(s, 3H); 5.59(s, 2H); 7.80(s 1H); 7.88(s, 1H); 9.36(s, $1 \mathrm{H}),{ }^{13} \mathrm{C}$ NMR $(150 \mathrm{MHz}$, temp. $298 \mathrm{~K}$, DMSO-d $\left._{6}, \mathrm{TMS}\right): \delta(\mathrm{ppm})=14.64 ; 35.96 ; 64.84$; $77.95 ; 121.93 ; 124.08 ; 137.31$.

3-(etoxymethyl)-1,2-dimethylimidazolium hydrogen sulfate(VI) (emd): ${ }^{1} \mathrm{H}$ NMR $(600 \mathrm{MHz}$, temp. $\left.298 \mathrm{~K}, \mathrm{DMSO}_{\mathrm{d}}, \mathrm{TMS}\right): \delta(\mathrm{ppm})=1.10-1.12(\mathrm{t}$, $\mathrm{J}=7 \mathrm{MHz}, 3 \mathrm{H}) ; 2.64(\mathrm{~s}, 3 \mathrm{H}) ; 3.52-3.53(\mathrm{~d}, 2 \mathrm{H})$; $3.80(\mathrm{~s}, 3 \mathrm{H}) ; 5.56(\mathrm{~s}, 2 \mathrm{H}) ; 7.69(\mathrm{~s} 1 \mathrm{H}) ; 7.79(\mathrm{~s}, 1 \mathrm{H}) .{ }^{13} \mathrm{C}$ NMR (150 MHz, temp. $298 \mathrm{~K}$, DMSO-d $_{6}$, TMS): $\delta$ $(\mathrm{ppm})=9.39 ; 14.67 ; 34.74 ; 64.40 ; 76.86 ; 121.19$; $122.41 ; 145.49$.

3-(benzyloxymethyl)-1-methylimidazolium hydrogen sulfate(VI) (bom): ${ }^{1} \mathrm{H}$ NMR (600 MHz, temp. $\left.298 \mathrm{~K}, \mathrm{DMSO}-\mathrm{d}_{6}, \mathrm{TMS}\right): \delta(\mathrm{ppm})=3.90(\mathrm{~s}, 3 \mathrm{H})$; 4.63(s, 2H); 5.70(s, 2H); 7.32-7.37(m, 5H); $7.78(\mathrm{~s}, 1 \mathrm{H}) ; 7.90(\mathrm{~s}, 1 \mathrm{H}) ; 9.38(\mathrm{~s}, 1 \mathrm{H}) .{ }^{13} \mathrm{C}$ NMR (150 MHz, temp, $298 \mathrm{~K}$, DMSO- $d_{6}$, TMS): $\delta(\mathrm{ppm})=$ $35.90 ; 71.01 ; 77.88 ; 121.91 ; 124.04 ; 127.81 ; 127.94$; $128.34 ; 136.60 ; 137.43$.

X-ray diffraction

Supermolecular structures were analyzed by means of powder X-ray diffraction (pXRD) using $\mathrm{CuK} \alpha$ radiation at $30 \mathrm{kV}$ and $25 \mathrm{~mA}$ anode excitation. The X-ray diffraction patterns were recorded for the angle range of from $5^{\circ}$ to $30^{\circ}$ in the step of $0.04^{\circ} / 3 \mathrm{~s}$. Deconvolution of peaks was performed by the method proposed by Hindeleh and Johnson (1971), improved and programmed by Rabiej (1991). The deconvolution of a sample cellulose diffraction pattern for crystallinity measurement is shown is Supplementary Material (Fig. S1). After separation of X-ray diffraction lines, the crystallinity index $\left(\mathrm{X}_{\mathrm{c}}\right)$ of cellulosic materials was calculated by comparison of areas under crystalline peaks and amorphous curve.

\section{FTIR analysis}

FTIR analysis was performed to determine changes in chemical structure of cellulose after ionic liquid treatment. FTIR spectra were recorded on an ATI Mattson Infinity Series FTIR spectrometer equipped with a deuterated triglycine sulfate detector, in range from 500 to $4000 \mathrm{~cm}^{-1}$.

FTIR-ATR analysis

Chitosan and composite samples were analyzed using a Fourier transform attenuated total reflectance (ATR) spectrometer Alpha (Bruker Optics GmbH). Spectra were recorded in range from 500 to $4000 \mathrm{~cm}^{-1}$. The spectral resolution of the spectrophotometer was $4 \mathrm{~cm}^{-1}$, each spectrum was computed as an average of 32 successive measurements in order to minimize the measurement error. Each sample was tested five times.

Elementary analysis

The analysis of carbon, hydrogen, nitrogen, and sulfur concentration in cellulosic materials was determined by the Thermo Scientific Flash 2000 CHNS/O Analyzer (Thermo Fisher Scientific, USA). Instrument was calibrated with the 2,5-bis-(5-tert-butyl-benzoxazol-2-yl)thiophene (Thermo Fisher Scientific, USA) and Birch Leaf (Elemental Microanalysis Ltd, UK) standards.

Particle size determination

Zetasizer Nano ZS (Malvern Instruments Ltd.) employing the laser diffraction technique in the range of $0.6-6000 \mathrm{~nm}$, respectively was applied to determine particle size and the dispersive properties of cellulosic materials. Before each test $0.01 \mathrm{~g}$ of tested material was dispersed in $25 \mathrm{~cm}^{3}$ of propanol and homogenized by ultrasonication for $20 \mathrm{~min}$.

Mechanical testing of composites

Tensile properties of composites were defined using Zwick and Roell Allround-Line Z020 TEW testing machine. Samples of $10 \mathrm{~mm}$ width and thickness ca. $0.1 \mathrm{~mm}$ were tested with speed $5 \mathrm{~mm} / \mathrm{min}$ and initial force $0.2 \mathrm{~N}$ in accordance to standard ISO 527-3. The arithmetic mean of at least seven replicate determinations was taken into consideration in each case. 


\section{Results and discussion}

Chemical structure and elementary composition of cellulose treated with ionic liquids

Figure 1 shows FTIR spectra of starting cellulose and cellulose hydrolyzed with ionic liquids. In accordance to the literature (Satyamurthy and Vigneshwaran 2013) all the characteristic bands of cellulosic materials were identified. All spectra contained bands coming from following bonds: $\mathrm{O}-\mathrm{H}\left(\sim 3400 \mathrm{~cm}^{-1}\right.$ and $\left.\sim 1650 \mathrm{~cm}^{-1}\right), \mathrm{C}-\mathrm{O}-\mathrm{O}\left(\sim 1730 \mathrm{~cm}^{-1}\right), \mathrm{C}-\mathrm{H}$ and $\mathrm{O}-\mathrm{CH}$ stretching $\left(\sim 1450 \mathrm{~cm}^{-1}\right), \mathrm{C}-\mathrm{H}$ bending $\left(\sim 1370 \mathrm{~cm}^{-1}\right), \mathrm{C}-\mathrm{O}-\mathrm{C}$ stretching $\left(\sim 1030 \mathrm{~cm}^{-1}\right)$, and $\mathrm{C}-\mathrm{OH}$ bending $\left(\sim 670 \mathrm{~cm}^{-1}\right)$. Samples treated with ionic liquid did not show any bands coming from imidazole ring of ionic liquids proving that produced celluloses were sufficiently washed.

The results of elementary analysis given in Table 2 prove that produced materials did not contain any significant ionic liquids residues. In micrometric cellulose I neither nitrogen nor sulfur was detected. Hydrolyzed samples were characterized with small content of nitrogen and sulfur, what is a result of hydrolysis process, remains of ionic liquid. Yang et al. (2006) performed studies in which the nitrogen content in Avicel PH-101 cellulose was found to be even higher $(0.40 \pm 0.02 \%)$ than in ionic liquid treated samples produced in this research.

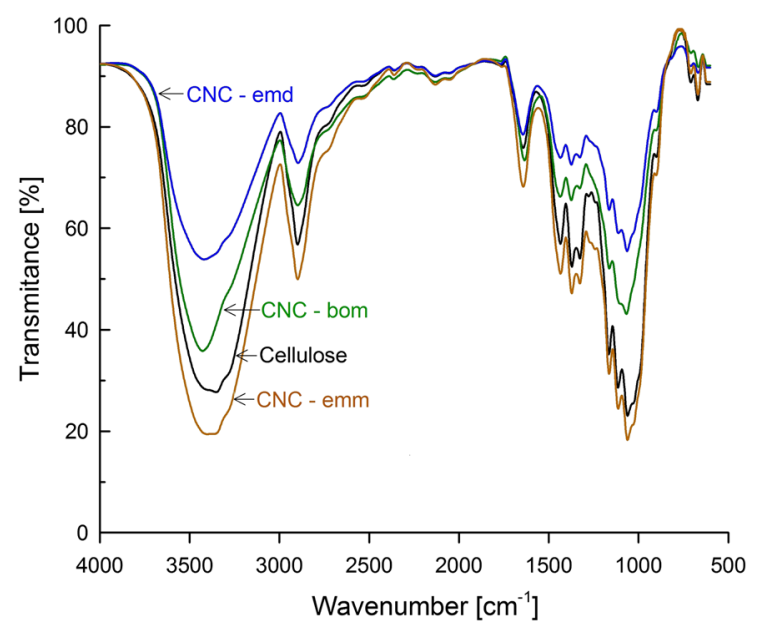

Fig. 1 FTIR spectra of cellulose I and celluloses hydrolyzed with ionic liquids
Supermolecular structure of celluloses

Cellulosic materials were analyzed in terms of supermolecular structure. In Fig. $2 \mathrm{X}$-ray diffraction patterns of cellulose and ionic liquid treated celluloses are shown. Figure S2 shows the diffraction patterns of the various composites.

Regardless of the ionic liquid used, the supermolecular structure of each sample was characteristic for cellulose I. There were no peaks coming from crystallographic structures of cellulose II proving that no polymorphic conversion occurred. In comparison with starting cellulose I, intensity of peaks recorded for samples CNC-emd and CNC-bom, treated with new ionic liquids was significantly increased. It is noticeable especially for peak at $2 \theta \approx 22.5^{\circ}$, coming from lattice plane (200). Changes in shape of diffractograms can be assigned to higher degree of order of hydrolyzed cellulose. Starting cellulose I consisted of $63 \%$ of crystalline phase, whereas degree of crystallinity of ionic liquid treated samples was in range from 68 to $77 \%$. The overall increase in crystallinity degree is in line with literature (Man et al. 2011; Kos et al. 2014). That proves that ionic liquid hydrolysis was effective, caused degradation of amorphous regions, and thus increased the content of crystalline phase.

Determination of particles size for ionic liquid treated celluloses

One of the most important aspects of this study was to find the relationship between the chemical structure of ionic liquid cation and size of the particles produced using this particular hydrolyzing agent. Figure 3 presents the findings of laser diffraction technique which enabled determination of particle size of celluloses treated with different ionic liquids.

It is clearly visible that the cation type used for treatment has a great influence on particle size of produced materials. Hydrolysis of cellulose I with ionic liquid named 'emm', with an oxygen atom in side chain of imidazole ring, was responsible for obtaining particles with wide distribution of diameters. Sample CNC-emm contained $56 \%$ of particles with diameters $\leq 220 \mathrm{~nm}$. Most probably these particles were prone to agglomeration and in result high amount of particles of size $\leq 2670 \mathrm{~nm}$ was observed. Similar results were observed by other authors 
Table 2 Content of nitrogen, carbon, hydrogen, and sulfur in untreated cellulose and in samples after ionic liquid treatment

nd not detected

\begin{tabular}{lllll}
\hline & $\mathrm{N}$ & $\mathrm{C}$ & $\mathrm{H}$ & $\mathrm{S}$ \\
\hline Content (\%) & & & & \\
Cellulose I & nd & $42.830 \pm 0.002$ & $6.364 \pm 0.050$ & nd \\
CNC-emm & $0.176 \pm 0.001$ & $41.848 \pm 0.006$ & $6.311 \pm 0.048$ & $0.246 \pm 0.025$ \\
CNC-emd & $0.159 \pm 0.003$ & $41.446 \pm 0.012$ & $6.214 \pm 0.056$ & $0.176 \pm 0.005$ \\
CNC-bom & $0.128 \pm 0.004$ & $42.344 \pm 0.009$ & $6.238 \pm 0.158$ & $0.114 \pm 0.001$ \\
\hline
\end{tabular}

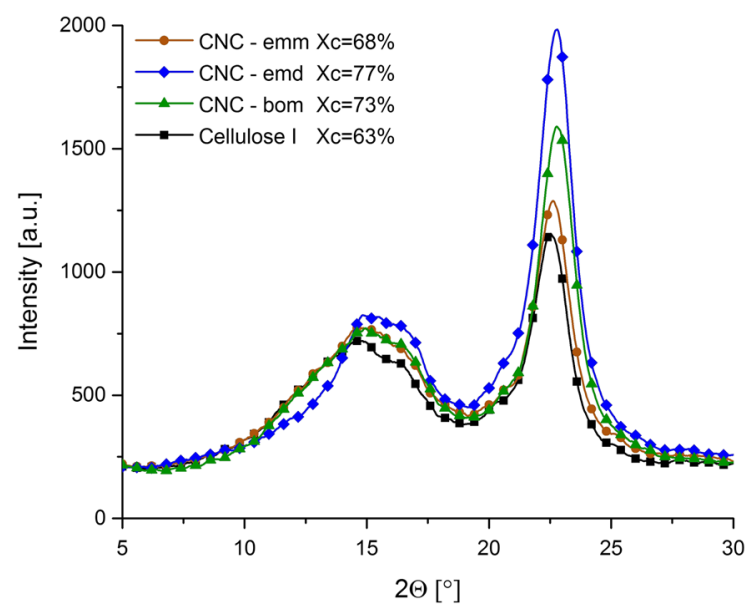

Fig. 2 Diffractograms of cellulose I and celluloses hydrolyzed with ionic liquids

(Dufresne 2013) who explain this behavior with presence of strong $\mathrm{O}-\mathrm{H}$ intermolecular bonds occurring in cellulose. Diameters of cellulose particles treated with 3-(etoxymethyl)-1,2-dimethylimidazolium hydrogen sulfate(VI) were definitely narrower. CNC-emd sample contained particles only in range from 91 to $342 \mathrm{~nm}$. Interestingly, the only difference in chemical structure of 'emm' and 'emd' ionic liquids was the presence of extra methyl group in the imidazole ring of 'emd'. It turns out that blockage of proton in $\mathrm{C} 2$ position of imidazole ring is responsible for significant reduction of dispersity of obtained particles. Cellulose hydrolyzed with 'bom' ionic liquid, containing in its structure a benzyl ring, was characterized with narrow and well-defined particle size distributions. This CNC-bom sample had diameters in range 164-342 $\mathrm{nm}$. What is important, in case of both CNC-emd and CNC-bom sample no micrometric particles were observed. However, it assumed that regardless of the hydrolyzing agent used particles of size $\leq 122 \mathrm{~nm}$ showed a tendency to agglomerate. Similar behavior was also observed in our previous studies regarding acid (Borysiak and Grząbka-Zasadzińska 2016) and 1-butyl-3-methylimidazolium hydrogen sulfate (Grząbka-Zasadzińska et al. 2017) hydrolysis of celluloses.

Chemical structure of chitosan/nanometric cellulose composites

The FTIR-ATR spectra of chitosan and its composites shown in Fig. 4 were recorded in order to determine
Fig. 3 Particle size distribution of cellulosic materials

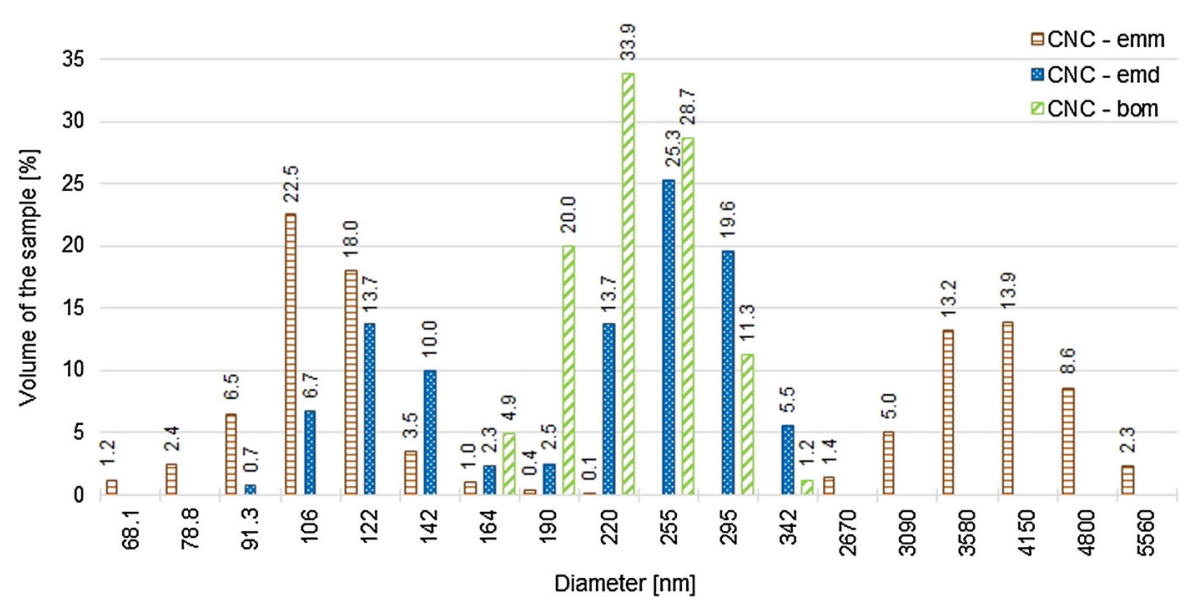


the infrared bands and shifts related to formation of CHT/CNC composites.

The position of the peaks of chitosan film in spectrum is similar to those described previously by numerous authors (Wang et al. 2005; Cao et al. 2007; Zhang et al. 2011). The absorption peaks of the chitosan films at $3500-3250 \mathrm{~cm}^{-1}$ can be assigned to the stretching of intra- and intermolecular $\mathrm{O}-\mathrm{H}$ and $\mathrm{CH}_{2} \mathrm{OH}$, overlapping with stretching $\mathrm{NH}_{2}$ (3500-3400 $\mathrm{cm}^{-1}$ ), and N-H secondary amides vibrations $\left(3300-3280 \mathrm{~cm}^{-1}\right)$. Peak at $2920-2870 \mathrm{~cm}^{-1}$ corresponds to symmetric and asymmetric $\mathrm{C}-\mathrm{H}$ vibrations. Bands coming from amide I, amide II, and amide III at $1640 \mathrm{~cm}^{-1}, 1549 \mathrm{~cm}^{-1}$, and $1261 \mathrm{~cm}^{-1}$, respectively have also been observed.

When compared to unfilled chitosan, spectra recorded for composites did not show major variations. That was due to the fact that both cellulose and chitosan are polysaccharides and their chemical structure, except for presence of the amide groups in chitosan, is very similar. Positions of bands characteristic for produced nanocelluloses were already given in "Chemical structure and elementary composition of cellulose treated with ionic liquids" section of this paper.

In spectra of composites (Fig. 4) all representative bands of chitosan and cellulose were present. However, it can be seen that incorporation of $5 \%$ of $\mathrm{CNC}$ in chitosan matrix resulted in some differences. Peak at $\sim 3340 \mathrm{~cm}^{-1}$ observed only for composite samples suggests occurrence of hydrogen bonding between $\mathrm{CNC}$ and polymer matrix (Khan et al. 2010, 2012). For composites absorption bands of $\sim 1200 \mathrm{~cm}^{-1}$ and $\sim 1549 \mathrm{~cm}^{-1}$ were slightly shifted to lower wavenumbers indicating that bonds for $\mathrm{CH}, \mathrm{NH}$, and $\mathrm{OH}$ groups are weaker and $\mathrm{C}, \mathrm{N}$, and $\mathrm{H}$ atoms are stronger attracted to other ambient atoms (Xu et al. 2018). Other changes resulting from addition of $\mathrm{CNC}$ were rather minor because of the low amount of $\mathrm{CNC}$ used to form composites.

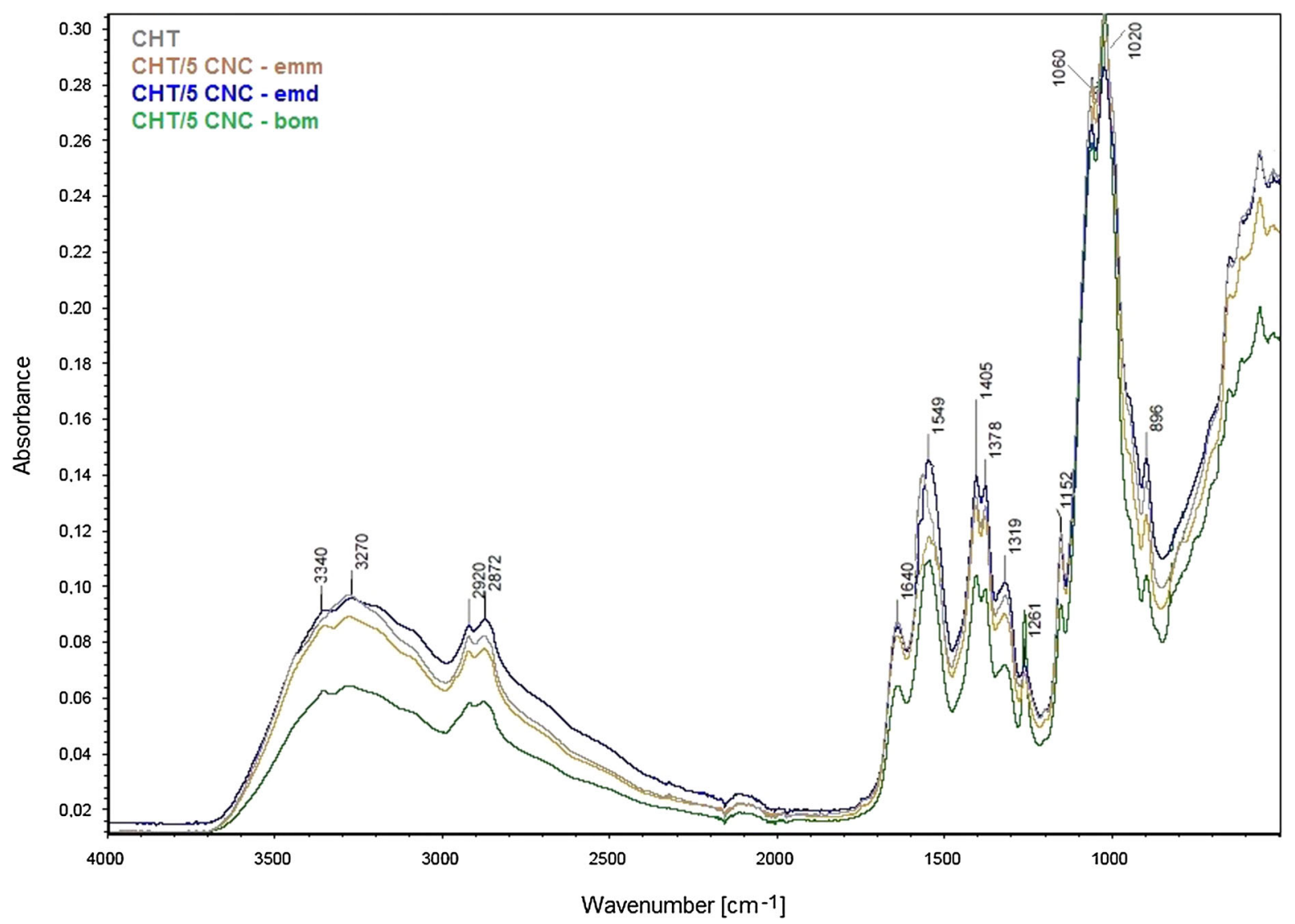

Fig. 4 FTIR-ATR spectra of chitosan and its composites 
Mechanical testing of chitosan/nanometric cellulose composites

Produced nanometric celluloses were incorporated in chitosan polymer matrix. Tensile parameters obtained during mechanical test of composites are given in Table 3.

In terms of Young's modulus incorporation of each type of the filler was responsible for increase of this parameter when compared to unfilled chitosan. Definitely the highest value of Young's modulus, $2453 \mathrm{MPa}$, was calculated for chitosan filled with $1 \%$ of CNC-bom filler. YM for composite with $3 \%$ of CNC-emm reached $1630 \mathrm{MPa}$, but if standard deviations are taken into consideration it turns out that values recorded for chitosan with $1 \%$ and $3 \%$ of CNCemm were rather comparable. YM for composites filled with $1 \%$ and $5 \%$ of CNC-emd was slightly lower, reaching $1557 \mathrm{MPa}$ and $1313 \mathrm{MPa}$, respectively.

Introduction into polymer matrix of $1 \%$ and $3 \%$ of cellulose hydrolyzed with 3-(etoxymethyl)-1methylimidazolium hydrogen sulfate(VI) resulted in over $30 \%$ increase of tensile strength when compared to pristine chitosan. For composite with $5 \%$ of this filler value of TS dropped to $21.2 \mathrm{MPa}$. In case of chitosan with $\mathrm{CNC}-$ emd the best results were obtained for composites with $1 \%$ of the filler, the TS reached the value of $60.1 \mathrm{MPa}$. Composites with $5 \%$ of this filler were characterized with lower, but still relatively high value of TS-35.3 MPa. Addition of 3\% of the CNC-

Table 3 Young's modulus (YM), tensile strength (TS), and elongation at break (EB) parameters obtained for tested specimens

\begin{tabular}{lccr}
\hline & YM $(\mathrm{MPa})$ & TS $(\mathrm{MPa})$ & \multicolumn{1}{c}{ EB $(\%)$} \\
\hline CHT & $236 \pm 56$ & $24.7 \pm 1.5$ & $41.9 \pm 4.8$ \\
CHT/1 CNC-emm & $1360 \pm 44$ & $32.5 \pm 3.9$ & $30.4 \pm 1.3$ \\
CHT/3 CNC-emm & $1630 \pm 261$ & $35.2 \pm 3.5$ & $33.9 \pm 3.2$ \\
CHT/5 CNC-emm & $759 \pm 128$ & $21.2 \pm 1.4$ & $5.8 \pm 0.9$ \\
CHT/1 CNC-emd & $1557 \pm 114$ & $60.1 \pm 4.8$ & $40.1 \pm 1.4$ \\
CHT/3 CNC-emd & $542 \pm 104$ & $16.9 \pm 1.2$ & $28.5 \pm 3.8$ \\
CHT/5 CNC-emd & $1313 \pm 29$ & $35.3 \pm 1.3$ & $9.8 \pm 2.3$ \\
CHT/1 CNC-bom & $2453 \pm 146$ & $64.9 \pm 7.1$ & $19.8 \pm 2.0$ \\
CHT/3 CNC-bom & $810 \pm 87$ & $26.0 \pm 3.3$ & $15.4 \pm 1.7$ \\
CHT/5 CNC-bom & $1263 \pm 132$ & $29.4 \pm 2.8$ & $6.2 \pm 1.6$ \\
\hline
\end{tabular}

emd filler to polymer matrix led to decrease of tensile strength to the lowest noted value, 16.9 MPa. The most significant increase in tensile strength, up to 64.9 MPa, was noted for chitosan with $1 \%$ of cellulose hydrolyzed with 3-(benzyloxymethyl)-1-methylimidazolium hydrogen sulfate(VI). As the amount of the CNC-bom filler in composite increased to $3 \%$ and 5\%, the TS parameter reached value of $26 \mathrm{MPa}$ and 29.4 MPa, respectively.

In terms of both, Young's modulus and tensile strength, the highest values were observed for composites with $1 \%$ loading of a filler. Also our previous research shows that $1 \%$ content of a nanocellulosic filler in chitosan matrix is optimal (Grząbka-Zasadzińska et al. 2018). This is consistent with other literature reports in which it was shown that composites with the smallest amount of filler are characterized by the highest values of strength parameters, resulting from better dispersion of fillers in the polymer matrix (Haafiz et al. 2013; Ambrosio-Martín et al. 2015). A similar phenomenon was observed by Boufi et al. (Boufi et al. 2014), who noted that the decrease in the TS value along with the increase in the content of nanometric cellulose in the polymer matrix results from the limitation of polymer chain movements. Surprisingly, in our study values registered for composites with $3 \%$ of each filler were the lowest. However, further increase of filler content up to 5\% resulted in improvement of TS and YM parameters. Decrease of YM and TS observed for materials with $3 \%$ of a filler can be assigned to aggregation of a filler. Tendency of filler to aggregate results from its high surface energy and high specific area of nanometric particles (Mendes et al. 2015; Reid et al. 2016). Moreover, during preparation of chitosan/CNC solutions, apart from aggregation, a competitive, reversible process of agglomeration takes place. If mechanical or ultrasonic homogenization is applied these agglomerates can be splitted into smaller particles (Seipenbusch et al. 2010).

Elasticity of composite films with $1 \%$ and $3 \%$ of CNC-emm filler was similar, ca. $30 \%$. Only the use of the maximum content of this filler resulted in a significant reduction in elasticity, to $\mathrm{EB}=5.8 \%$. Chitosan with $1 \%$ CNC-emd was characterized with the highest value of $\mathrm{EB}=40.1 \%$, which is comparable to reference sample of chitosan $(\mathrm{EB}=41.9 \%)$. This is rather exceptional, because in among all other composites, a considerable decrease of elongation at break, 
typical for nanocomposites, was observed. Increase of the CNC-emd filler content only to $3 \%$ led to lowering the elasticity to $28.5 \%$, whereas for chitosan/5 CNCemd the EB was found to be only $9.8 \%$. Similar behavior was noted also for films with the last type of filler, CNC-bom. The EB parameter for chitosan with maximum loading of this filler was $6.2 \%$.

In view of the above, it turns out that not only particle size of filler, but also it homogeneity had an important influence on mechanical properties of composites.

The highest values of elongation at break were registered for films with $1 \%$ and $3 \%$ of $\mathrm{CNC}-\mathrm{emm}$ and CNC-emd fillers which contained particles $\leq 122 \mathrm{~nm}$. Filler produced using 3-(benzyloxymethyl)-1-methylimidazolium hydrogen sulfate(VI) was characterized with narrow particle size distribution, but at the same time did not have particles with diameter $\leq 122 \mathrm{~nm}$. In result, composites with this CNC-bom filler were definitely less elastic that other films. To sum up, elongation at break parameter was influenced by the presence of micrometric particles and particles with nanometric size $(\leq 122 \mathrm{~nm})$.Both types of composites that did not contain micrometric particles (with $\mathrm{CNC}-\mathrm{emd}$ and CNC-bom) are characterized with different elasticity which results from presence of particles with size $\leq 122 \mathrm{~nm}$.

Homogeneity of filler particle size was found to be crucial in terms of values of Young's modulus. Definitely the highest YM was noted for chitosan with $1 \%$ of CNC-bom filler, whose all particles were in range between 164 and $342 \mathrm{~nm}$. Lower values of YM were obtained for composites with $1 \%$ of CNCemd (contained particles smaller than $164 \mathrm{~nm}$ and no micrometric agglomerates). The lowest YM was observed for films with CNC-emm filler that had two main fractions of particles-with diameters $\leq 122 \mathrm{~nm}$ and micrometric agglomerates over $2670 \mathrm{~nm}$.

Particle size distribution was found to influence not only Young's modulus but also tensile strength of studied composites. Similarly, the highest value of TS (64.9 MPa for $1 \%$ of filler) was calculated for films with CNC-bom filler which was characterized with the narrowest range of particle diameters (Fig. 5). Composites with CNC-emd filler, that in comparison with CNC-bom contained particles $\leq 122 \mathrm{~nm}$, resulted in obtaining lower, but still high values of
TS (60.1 MPa for $1 \%$ loading). Introduction into chitosan matrix CNC-emm filler, with high amount of both, micrometric and nanometric particles, resulted in the lowest values of tensile strength.

Analysis of mechanical tests results of composites in relation to the distribution of particles size of fillers allowed to determine that the key factor determining the mechanical properties was application of a filler with a specific distribution of particle size. When comparing composites with $1 \%$ of filler it can be seen that the highest values of Young's modulus were obtained using filler with the lowest dispersion of particle size, while maximum tensile strength was recorded for composites with filler that did not contain micrometric particles. Composite materials with high elasticity were produced by incorporating into chitosan filler that had particles with diameters $\leq 122 \mathrm{~nm}$. Surprisingly, particles size of the filler is not the only aspect determining mechanical properties of the composites. The key factor was found to be dispersity of produced cellulose particles. High amount of nanoparticles did not necessarily resulted in obtaining optimal mechanical properties. Composites with high values of tensile strength and elasticity were produced if filler with low dispersity of particle size was used, even if particles larger than $100 \mathrm{~nm}$ were present. Based on conducted research, it was shown that designing a cation of ionic liquid with an appropriate size could enable the control of the size of cellulose nanoparticles and, as in consequence, the mechanical properties of the composites.

\section{Conclusions}

In this paper the influence of the cation type of newly synthesized imidazolium ionic liquids on distribution of particle size was determined. The cation with the aliphatic substituent was responsible for the formation of particles with a size of $\leq 122 \mathrm{~nm}$. The use of an ionic liquid with a cation containing a benzyl substituent was responsible for obtaining filler with narrow dispersity range of particle size. It was found that type of the ionic liquid used has also an influence on crystallinity degree of the produced materials.

Incorporation into chitosan matrix filler hydrolyzed with cation containing benzyl substituent (characterized with narrow dispersity of particle size) resulted in obtaining materials with high values of Young's 
Fig. 5 Relationship between tensile strength of composites with $1 \%$ of the filler and particle size distribution of each filler

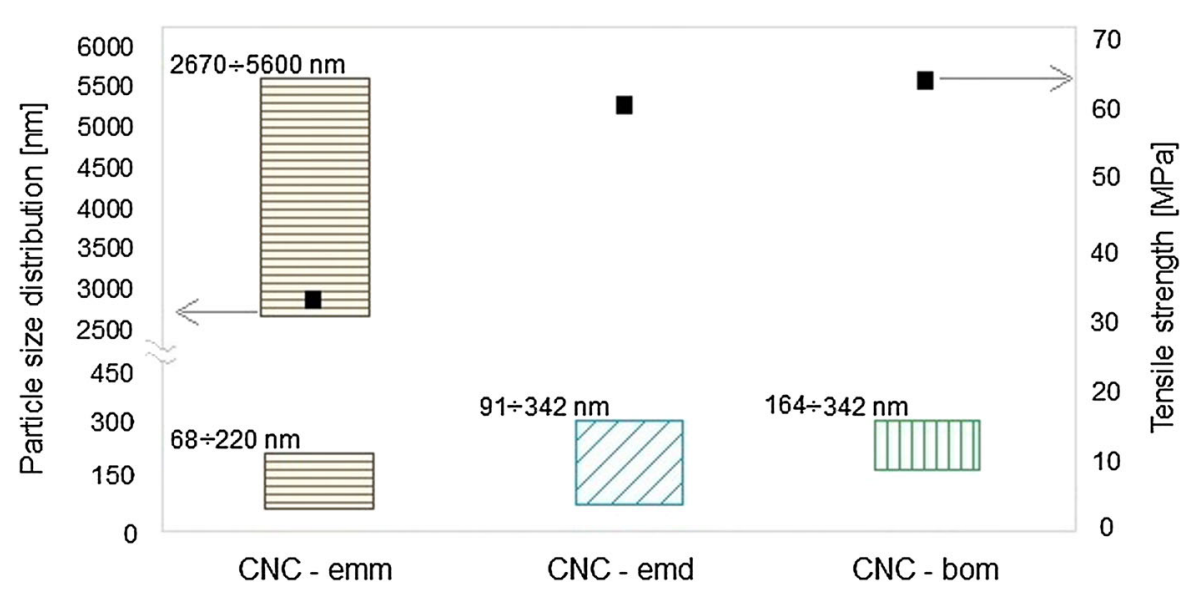

References

modulus. Composites with high values of tensile strength were produced if used filler did not contain particles larger than $2 \mu \mathrm{m}$. Such fillers, with particle diameters $\leq 342 \mathrm{~nm}$, were produced using ionic liquids with 3-(etoxymethyl)-1-methylimidazolium and 3-(benzyloxymethyl)-1-methylimidazolium cations. Therefore, the narrower was the particle size distribution, the higher the values of tensile strength of composites were recorded. Introduction of filler with diameters $\leq 122 \mathrm{~nm}$ and no micrometric particlescellulose hydrolyzed with 3-(etoxymethyl)-1,2dimethylimidazolium hydrogen sulfate(VI)—resulted in preparation of films with high elongations at break.

The obtained results should be important in terms of designing properties of composite materials. Finding a yet unrecorded relationship between dispersive characteristics of nanometric celluloses and mechanical properties of chitosan/nanometric cellulose composites enables formation of composites with assumed macroscopic features.

Acknowledgments This work was supported by the Polish Ministry of Science and Higher Education, Grant No 03/32/ SBAD/0903.

Open Access This article is distributed under the terms of the Creative Commons Attribution 4.0 International License (http:// creativecommons.org/licenses/by/4.0/), which permits unrestricted use, distribution, and reproduction in any medium, provided you give appropriate credit to the original author(s) and the source, provide a link to the Creative Commons license, and indicate if changes were made.
Ambrosio-Martín J, Lopez-Rubio A, Fabra MJ, Gorrasi G, Pantani R, Lagaron JM (2015) Assessment of ball milling nanocrystals nanocomposites. J Appl Polym Sci. https:// doi.org/10.1002/app.41605

Borysiak S, Grząbka-Zasadzińska A (2016) Influence of the tals and their application in chitosan/nanocellulose composites. J Appl Polym Sci 1:133. https://doi.org/10.1002/ app.42864

Borysiak S, Grząbka-Zasadzińska A, Odalanowska M, Skrzypczak A, Ratajczak I (2018) The effect of chemical modification of wood in ionic liquids on the supermolecular structure and mechanical properties of wood/ polypropylene composites. Cellulose 25:4639-4652. https://doi.org/10.1007/s10570-018-1892-2

Boufi S, Kaddami H, Dufresne A (2014) Mechanical performance and transparency of nanocellulose reinforced polymer nanocomposites. Macromol Mater Eng 299:560-568. https://doi.org/10.1002/mame.201300232

Brandt A, Grasvik J, Hallett JP, Welton T (2013) Deconstruction of lignocellulosic biomass with ionic liquids. Green Chem 15:550-583. https://doi.org/10.1039/C2GC36364J

Cao X, Dong H, Li CM (2007) New nanocomposite materials reinforced with flax cellulose nanocrystals in waterborne polyurethane. Biomacromolecules 8:899-904. https://doi. org/10.1021/bm0610368

Dehnad D, Mirzaei H, Emam-Djomeh Z, Jafari S-M, Dadashi S (2014) Thermal and antimicrobial properties of chitosannanocellulose films for extending shelf life of ground meat. Carbohyd Polym 109:148-154. https://doi.org/10.1016/j. carbpol.2014.03.063

Dufresne A (2013) Nanocellulose: a new ageless bionanomaterial. Mater Today 16:220-227. https://doi.org/10.1016/j. mattod.2013.06.004

Falamarzpour P, Behzad T, Zamani A (2017) Preparation of nanocellulose reinforced chitosan films, cross-linked by adipic acid. Int J Mol Sci 18:396

Fernandes SCM, Oliveira L, Freire CSR, Silvestre AJD, Neto CP, Gandini A, Desbriéres J (2009) Novel transparent methodology to develop polylactide-bacterial cellulose polymorphism of cellulose on the formation of nanocrys- 
nanocomposite films based on chitosan and bacterial cellulose. Green Chem 11:2023-2029. https://doi.org/10. 1039/b919112g

Fernandes SCM, Freire CSR, Silvestre AJD, Pascoal Neto C, Gandini A (2011) Novel materials based on chitosan and cellulose. Polym Int 60:875-882. https://doi.org/10.1002/ pi. 3024

Ghasemi M, Alexandridis P, Tsianou M (2017) Cellulose dissolution: insights on the contributions of solvent-induced decrystallization and chain disentanglement. Cellulose 24:571-590. https://doi.org/10.1007/s10570-016-1145-1

Grząbka-Zasadzińska A, Amietszajew T, Borysiak S (2017) Thermal and mechanical properties of chitosan nanocomposites with cellulose modified in ionic liquids. J Therm Anal Calorim 130:1-12. https://doi.org/10.1007/s10973017-6295-3

Grząbka-Zasadzińska A, Smułek W, Kaczorek E, Borysiak S (2018) Chitosan biocomposites with enzymatically produced nanocrystalline cellulose. Polym Composite 39:E448-E456. https://doi.org/10.1002/pc.24552

Haafiz MKM, Hassan A, Zakaria Z, Inuwa IM, Islam MS, Jawaid M (2013) Properties of polylactic acid composites reinforced with oil palm biomass microcrystalline cellulose. Carbohyd Polym 98:139-145. https://doi.org/10. 1016/j.carbpol.2013.05.069

Hindeleh AM, Johnson DJ (1971) The resolution of multipeak data in fibre science. J Phys D Appl Phys 4:259

HPS AK et al (2016) A review on chitosan-cellulose blends and nanocellulose reinforced chitosan biocomposites: properties and their applications. Carbohyd Polym 150:216-226. https://doi.org/10.1016/j.carbpol.2016.05.028

Isik M, Sardon H, Mecerreyes D (2014) Ionic liquids and cellulose: dissolution, chemical modification and preparation of new cellulosic materials. Int J Mol Sci 15:11922

Jonoobi M, Oladi R, Davoudpour Y, Oksman K, Dufresne A, Hamzeh Y, Davoodi R (2015) Different preparation methods and properties of nanostructured cellulose from various natural resources and residues: a review. Cellulose 22:935-969. https://doi.org/10.1007/s10570-015-0551-0

Khan RA, Salmieri S, Dussault D, Uribe-Calderon J, Kamal MR, Safrany A, Lacroix M (2010) Production and properties of nanocellulose-reinforced methylcellulose-based biodegradable films. J Agric Food Chem 58:7878-7885. https://doi.org/10.1021/jf1006853

Khan A et al (2012) Mechanical and barrier properties of nanocrystalline cellulose reinforced chitosan based nanocomposite films. Carbohyd Polym 90:1601-1608. https://doi.org/10.1016/j.carbpol.2012.07.037

Kos T, Anžlovar A, Kunaver M, Huskić M, Žagar E (2014) Fast preparation of nanocrystalline cellulose by microwaveassisted hydrolysis. Cellulose 21:2579-2585. https://doi. org/10.1007/s10570-014-0315-2

Li H, Du Y, Xu Y (2004) Adsorption and complexation of chitosan wet-end additives in papermaking systems. J Appl Polym Sci 91:2642-2648. https://doi.org/10.1002/app. 13444

Li Y, Liu X, Zhang S, Yao Y, Yao X, Xu J, Lu X (2015) Dissolving process of a cellulose bunch in ionic liquids: a molecular dynamics study. Phys Chem Chem Phys 17:17894-17905. https://doi.org/10.1039/C5CP02009C
Lindman B, Karlström G, Stigsson L (2010) On the mechanism of dissolution of cellulose. J Mol Liq 156:76-81. https:// doi.org/10.1016/j.molliq.2010.04.016

Liu H, Sale KL, Holmes BM, Simmons BA, Singh S (2010) Understanding the interactions of cellulose with ionic liquids: a molecular dynamics study. J Phys Chem B 114:4293-4301. https://doi.org/10.1021/jp9117437

Man Z, Muhammad N, Sarwono A, Bustam MA, Vignesh Kumar M, Rafiq S (2011) Preparation of cellulose nanocrystals using an ionic liquid. J Polym Environ 19:726-731. https://doi.org/10.1007/s10924-011-0323-3

Mao J, Heck B, Reiter G, Laborie MP (2015) Cellulose nanocrystals' production in near theoretical yields by 1-butyl-3-methylimidazolium hydrogen sulfate ([Bmim] $\mathrm{HSO}_{4}$ )-Mediated hydrolysis. Carbohyd Polym 117:443-451. https://doi.org/10.1016/j.carbpol.2014.10. 001

Mendes CAdC, Ferreira NMS, Furtado CRG, de Sousa AMF (2015) Isolation and characterization of nanocrystalline cellulose from corn husk. Mater Lett 148:26-29. https:// doi.org/10.1016/j.matlet.2015.02.047

Nandi S, Guha P (2018) A review on preparation and properties of cellulose nanocrystal-incorporated natural biopolymer. J Package Technol Res 2:149-166. https://doi.org/10.1007/ s41783-018-0036-3

Pradhan S, Lach R, Le HH, Grellmann W, Radusch H-J, Adhikari R (2013) Effect of filler dimensionality on mechanical properties of nanofiller reinforced polyolefin elastomers. ISRN Polym Sci 2013:9. https://doi.org/10. 1155/2013/284504

Rabiej S (1991) A comparison of two X-ray diffraction procedures for crystallinity determination. Eur Polym J 27:947-954. 3057(91)90038-P

Rad-Moghadam K, Dehghan N (2014) Application of cellulose/chitosan grafted nano-magnetites as efficient and recyclable catalysts for selective synthesis of 3-indolylindolin-2-ones. J Mol Catal A Chem 392:97-104. https://doi. org/10.1016/j.molcata.2014.05.005

Reid MS, Villalobos M, Cranston ED (2016) Cellulose nanocrystal interactions probed by thin film swelling to predict dispersibility. Nanoscale 8:12247-12257. https:// doi.org/10.1039/C6NR01737A

Remsing RC, Swatloski RP, Rogers RD, Moyna G (2006) Mechanism of cellulose dissolution in the ionic liquid 1-nbutyl-3-methylimidazolium chloride: a $13 \mathrm{C}$ and $35 / 37 \mathrm{Cl}$ NMR relaxation study on model systems. Chem Commun. https://doi.org/10.1039/b600586c

Rubentheren V, Ward TA, Chee CY, Nair P, Salami E, Fearday C (2016) Effects of heat treatment on chitosan nanocomposite film reinforced with nanocrystalline cellulose and tannic acid. Carbohyd Polym 140:202-208. https://doi.org/ 10.1016/j.carbpol.2015.12.068

Satyamurthy P, Vigneshwaran N (2013) A novel process for synthesis of spherical nanocellulose by controlled hydrolysis of microcrystalline cellulose using anaerobic microbial consortium. Enzyme Microb Technol 52:20-25. https://doi.org/10.1016/j.enzmictec.2012.09.002

Seipenbusch M, Rothenbacher S, Kirchhoff M, Schmid H-J, Kasper G, Weber AP (2010) Interparticle forces in silica 
nanoparticle agglomerates. J Nanopart Res 12:2037-2044. https://doi.org/10.1007/s11051-009-9760-5

Suzuki T, Kono K, Shimomura K, Minami H (2014) Preparation of cellulose particles using an ionic liquid. J Colloid Interface Sci 418:126-131. https://doi.org/10.1016/j.jcis. 2013.12.014

Swatloski RP, Spear SK, Holbrey JD, Rogers RD (2002) Dissolution of cellose with ionic liquids. J Am Chem Soc 124:4974-4975. https://doi.org/10.1021/ja025790m

Trache D, Hussin MH, Haafiz MKM, Thakur VK (2017) Recent progress in cellulose nanocrystals: sources and production. Nanoscale 9:1763-1786. https://doi.org/10.1039/ C6NR09494E

Urbanik E, Zabielska-Matejuk J, Skrzypczak A (1999) Quantitative relation between surface active properties and antifungal activity of quaternary imidazolium chlorides (new wood preservatives). For Prod J 49:53-58

Wang S-F, Shen L, Zhang W-D, Tong Y-J (2005) Preparation and mechanical properties of chitosan/carbon nanotubes composites. Biomacromolecules 6:3067-3072. https://doi. org/10.1021/bm050378v

Xu K, Liu C, Kang K, Zheng Z, Wang S, Tang Z, Yang W (2018) Isolation of nanocrystalline cellulose from rice straw and preparation of its biocomposites with chitosan: physicochemical characterization and evaluation of interfacial compatibility. Compos Sci Technol 154:8-17. https://doi.org/10.1016/j.compscitech.2017.10.022

Yang B, Willies DM, Wyman CE (2006) Changes in the enzymatic hydrolysis rate of avicel cellulose with conversion. Biotechnol Bioeng 94:1122-1128. https://doi.org/ 10.1002/bit.20942

Youngs TGA et al (2011) Neutron diffraction, NMR and molecular dynamics study of glucose dissolved in the ionic liquid 1-ethyl-3-methylimidazolium acetate. Chem Sci 2:1594-1605. https://doi.org/10.1039/c1sc00241d

Zhang Z, Jiang T, Ma K, Cai X, Zhou Y, Wang Y (2011) Low temperature electrophoretic deposition of porous chitosan/ silk fibroin composite coating for titanium biofunctionalization. J Mater Chem 21:7705-7713. https://doi.org/10. 1039/C0JM04164E

Publisher's Note Springer Nature remains neutral with regard to jurisdictional claims in published maps and institutional affiliations. 